\title{
3 Research Square

\section{The Expression of YKL-40 in Peripheral Blood of Colorectal Cancer and Its Effect on the Proliferation and Angiogenesis of Colorectal Cancer Cells}

Shaohui Yang ( $\nabla$ doctoryang1980@163.com )

Ningbo Medical Treatment Centre Li Huili Hospital https://orcid.org/0000-0001-6543-8175

Jie Shen

Ningbo Medical Treatment Centre Li Huili Hospital

Yibin Zhao

Ningbo Medical Treatment Centre Li Huili Hospital

\section{Research Article}

Keywords: Colorectal Cancer, YKL-40, KeyGEN biotech

Posted Date: November 30th, 2021

DOl: https://doi.org/10.21203/rs.3.rs-1089158/v1

License: (c) (1) This work is licensed under a Creative Commons Attribution 4.0 International License.

Read Full License 


\section{Abstract}

Objective To observe the expression of YKL-40 in the serum of patients with colorectal cancer, and to study the effect of YKL-40 gene on the proliferation and angiogenesis of colon cancer cell lines.

Methods The serum of patients with colorectal cancer, precancerous lesions, and healthy controls were collected, and the expression of YKL-40 was detected by enzyme-linked immunosorbent assay (ELISA) technology. Screened cell lines with high expression of YKL-40 from colon cancer cell lines HCT-15, HCT116 , SW480, interfered with YKL-40 gene expression through siRNA technology, and co-cultured with bevacizumab, and detected cells with CCK8 method Proliferation, cell formation test to detect blood vessel formation.

Results The mean values of serum YKL-40 in the colorectal cancer group, precancerous lesion group and control group were $(178.50 \pm 71.91) \mu \mathrm{g} / \mathrm{L},(91.37 \pm 35.79) \mu \mathrm{g} / \mathrm{L}$ and $(78.23 \pm 26.52) \mu \mathrm{g} / \mathrm{L}$, respectively. The colorectal cancer group (preoperative) was significantly higher than the precancerous lesion group and the control group $(\mathrm{P}<0.01)$. The precancerous lesion group was higher than the control group, but the difference between the two was not statistically significant $(P=0.244)$. The expression of YKL-40 was positively correlated with the stage of colorectal cancer $(P<0.05)$. There was no significant difference in the expression of serum YKL-40 before and after surgery $(p=0.07)$. HCT-116 is a YKL-40 highly expressing cell line. After inhibiting the expression of this gene, the survival rate of the experimental group was $78.75 \%$, which was significantly lower than that of the control group $(p<0.05)$. The angiogenesis test is used to detect the angiogenesis ability. siRNA interference with YKL-40 gene and the addition of bevacizumab can inhibit the angiogenesis ability in vitro. Moreover,the vascular inhibitory effect of bevacizumab in the experimental group was stronger than that in the control group.

Conclusion YKL-40 is highly expressed in the peripheral blood of patients with colorectal cancer and is related to the tumor stage. The HCT-116 colon cancer cell line has a high expression of YKL-40. Interfering with the expression of YKL-40 can inhibit cell proliferation and angiogenesis. It suggests that YKL-40 plays an important role in the occurrence and development of colorectal cancer.

\section{Introduction}

Colorectal cancer (CRC) is known as the third most common cancer and the fourth most deadly cancer worldwide [1, 2]. It accounts for approximately $10 \%$ of all new cancer cases globally, remaining the second most frequent cause of cancer-related deaths [3,4]. As Western diets and lifestyles have had an increasing impact on human health, a rise in CRC incidence rates has followed $[5,6]$. In developing countries such as Argentina, Brazil, and China, the incidence and mortality rate due to CRC increased around $20 \%$ [7].

CRC is a silent disease, developing as a slow, multi-step process, that takes approximately $5-10$ years from premalignant lesions to CRC [8]. while up to $70 \%$ of cancers presenting with symptoms are at an advanced stage [9]. therefore, understanding the changes in genes related to the development of 
colorectal cancer and their molecular mechanisms are of great significance for improve prognosis through early detection and effective treatment. The YKL-40 gene is located on chromosome 1q32.1 [10] and encodes a highly conserved glycoprotein that combines heparin and chitin.Although the biological function of YKL-40 protein is not yet well understood, a large number of studies have shown that the protein encoded by YKL-40 has important functions in cell proliferation, differentiation, survival, and tissue remodeling. Ringsholt $\mathrm{M}$ et al. [11] found that YKL-40 protein was only highly expressed in embryonic or adult cells with high activity. Recent studies [12-14] found that YKL-40 is highly expressed in peripheral blood of patients with various human malignant tumors, suggesting that it is involved in the pathogenesis of various human tumors and is a new cancer-promoting gene. consequently, YKL-40 maybe recognized as a new prognostic and predictive marker in carcinomas. Moreover, YKL-40 plays a potential role in promoting tumor growth, which indicates that YKL-40 may serve as a therapeutic target. A mouse monoclonal anti-YKL-40 antibody ( $\mathrm{mAY}$ ) has shown to have therapeutic use in the treatment of tumor angiogenesis and metastasis [15]. The conjunctive therapy with $\mathrm{mAY}$ and ionizing irradiation (IR) synergistically inhibited tumor vascularization and progression in xenograft brain tumor models [16].In this study, the expression of YKL-40 in peripheral blood of patients with colorectal cancer was observed. And we screened out cell lines with high expression of YKL-40 gene. By silencing siRNA YKL-40, we compared the differences in cell activity and angiogenesis between the YKL-40 gene high expression group and the low expression group, and compare the response of the two groups to bevacizumab intervention. To explore the role of YKL-40 in the development of colorectal cancer and its feasibility as a biomarker for anti-angiogenesis therapy.

\section{Materials And Methods}

Clinical data: the study was approved by the ethics committee of Lihuili Hospital of Ningbo Medical Center (ethics approval No. ky2019pj035). Serum samples and clinicopathological data were collected from patients with colorectal cancer or precancerosis and healthy controls in LiHuili Hospital of Ningbo medical center. All patients were confirmed by pathology. The clinicopathological variables of subjects and the expression level of YKL-40 were summarized in Table 1.

Table 1 The correlation of YKL-40 expression with clinicopathologic characteristics of subjects 


\begin{tabular}{|c|c|c|c|c|c|}
\hline \multirow[t]{2}{*}{ group } & \multirow[t]{2}{*}{ Cases (n) } & \multirow[t]{2}{*}{ Media Age (y) } & \multicolumn{2}{|c|}{ Gender } & \multirow{2}{*}{$\begin{array}{l}\text { Ykl-40 expression } \\
\text { mean } \pm S D(u g / L)\end{array}$} \\
\hline & & & Male & Female & \\
\hline Controls & 21 & $40(18-72)$ & 11 & 10 & $78.23 \pm 26.52$ \\
\hline Precancerosis & 13 & $70(48-82)$ & 7 & 6 & $91.37 \pm 35.79$ \\
\hline Cancer & 79 & $64(25-89)$ & 49 & 30 & $178.50 \pm 71.91$ \\
\hline \multicolumn{2}{|l|}{ Differentiation } & & & & \\
\hline Well & 48 & & & & $165.26 \pm 63.08$ \\
\hline Poor & 28 & & & & $186.85 \pm 79.94$ \\
\hline Unknown & 3 & & & & $312.35 \pm 54.53$ \\
\hline Stage & 15 & & & & \\
\hline I & 18 & & & & $110.93 \pm 47.09$ \\
\hline II & 30 & & & & $145.35 \pm 46.69$ \\
\hline III & 16 & & & & $193.30 \pm 55.71$ \\
\hline IV & & & & & $251.38 \pm 62.81$ \\
\hline
\end{tabular}

Reagents and cell lines

HCT-15, HCT-116, and SW480 cells were all purchased from Beina Biological Technology Co., RIPA Pyrolysis liquid (Applygen), BCA Protein Assay Kit, RNA extraction kit, Trizon Reagent and RNA extraction kit were all purchased from Cwbiotech, PAGE Pre-Solution(A1010,Solarbio), Marker(Thermo), Mouse Monoclonal Anti-GAPDH, Horseradish enzyme-labeled goat anti-mouse $\lg \mathrm{G}(\mathrm{H}+\mathrm{L})$ and Horseradish Enzyme-labeled Goat Anti-Rabbit IgG $(\mathrm{H}+\mathrm{L})$ were all purchased from Zsbio, Rabbit Anti YKL-40 (Affinity),DMEM medium (KeyGEN biotech), CCK8 Cell proliferation assay Kit (KeyGEN biotech),FBS(BI), Trypsin-EDTA digestive fluid(Solarbio);Lipofectamine ${ }^{\text {TM }} 3000$ Transfection Reagent (Invitrogen), OPTI$M E M \otimes I$ reduced serum medium(Gibco) $₫ 5 / 5000$ Bevacizumab(Roche) $₫$ HiScript II Q RT SuperMix for qPCR (Vazyme) $₫ 2 \times S Y B R$ Green PCR Master Mix(Lifeint).

Enzyme linked immunosorbent assay (ELISA): Blood samples were collected before any treatment, centrifuged at $3000 \mathrm{rpm}$ for 10 minutes at room temperature, took the supernatant and stored at $-80{ }^{\circ} \mathrm{C}$ for standby. The serum concentration of YKL-40 was determined by ELISA. The kit was purchased from quidel company in the United States. According to the instructions, add 50ul standard or sample diluted 5 times to the enzyme label coated micropore, incubate at $37^{\circ} \mathrm{C}$ for 30 minutes, add 50 ul enzyme label reagent, and incubate at $37^{\circ} \mathrm{C}$ for 30 minutes. Add the developer and develop the color at $37^{\circ} \mathrm{C}$ for 10 minutes without light. After adding the termination solution, measure its absorbance at $450 \mathrm{~nm}$, draw the standard curve according to the relationship between the concentration and absorbance of the standard, 
calculate the regression equation, and then calculate the YKL-40 concentration according to the absorbance of the measured serum sample.

Cell culture: colon cancer cell lines HCT-15, HCT-116 and SW480 were cultured in DMEM medium containing $10 \%$ fetal bovine serum and placed in an incubator at $37^{\circ} \mathrm{C}$ and $5 \% \mathrm{CO} 2$. They were digested and subcultured with $0.25 \%$ trypsin every $2 \sim 3$ days according to the cell growth; The expression of YKL40 protein was detected by Western blotting (WB). According to the results, the cell line HCT116 with high expression of YKL-40 was selected as the follow-up experimental object.

Cell transfection: HCT116 cells were divided into two groups. One group was the control group and continued to be cultured in the original way; One group was the experimental group, and cell transfection was carried out: the cell culture medium was replaced with fresh culture medium, with a volume of $1 \mathrm{ml}$. Take 2 sterilized EP tubes, add 125ul opti MEM to each tube, add 5ul Lipofectamine 3000 to one tube, add 0.1 od plasmid and 5ul p3000 to the other EP tube, mix well and incubate at room temperature for 5min. The primers are shown in Table 1. Mix the above two EP tubes evenly, incubate at room temperature for $15 \mathrm{~min}$, drop the mixed solution into the corresponding hole in the six well plate, and put the cells back into the incubator for culture; Four hours after transfection, $1 \mathrm{ml}$ of fresh medium was added to the six well plate; After 48h, qPCR and WB verification were performed. The upstream primer of qPCR was 5 '- gtctgtcggaggatggaatggaact-3', and the downstream primer was 5 '- gat tag gtg gta aaa tgc tgt-3' $\beta$ - Actin is an internal reference, the upstream primer is 5 '- tgg cac cca gca caa tga a-3', and the downstream primer is 5 '- cta agt cag att ccg cta gag aag ca-3'. According to the verification results, the cell group with the best interference effect was selected for follow-up experiments.

Tab.2 siRNA primer sequences

SiRNA

SiRNA primer

SiRNA-1

F: 5'-CCA CAU CAU CUA CAG CUU UTT-3'

R: 5'-AAA GCU GUA GAU GAU GUG GTT-3'

SiRNA-2

F: 5'-GCA GCU AUG ACA UUG CCA ATT-3'

R: 5'-UUG GCA AUG UCA UAG CUG CTT-3'

SiRNA-3

F: 5'-GCA ACC AGU GGG UAG GAU ATT-3'

R: 5'-UAU CCU ACC CAC UGG UUG CTT-3'

Bevacizumab intervention and planking: the experimental group and the control group were further divided into the following groups: experimental group, experimental group + bevacizumab (10ug / $\mathrm{ml}$ ) culture group; Control group, control group + bevacizumab (10ug / ml) culture group, control group + empty group, each group continued to cultivate for $48 \mathrm{~h}$. After that, the cells were diluted according to the 
experimental needs, evenly spread into the cell culture plate, marked, placed in the incubator for culture, and the experiment was carried out after the cells completely adhered to the wall.

Detection of cell proliferation by CCK8 method: the cells in each group were diluted to 10 / UL, added to 96 well plates, 100 ul cell suspension was added to each well, $10 \mathrm{ul} \mathrm{CCK-8}$ was added after 24 hours of culture, and incubated at $37^{\circ} \mathrm{C}$ for 2 hours. The $450 \mathrm{~nm}$ absorbance value in 96 well plates was detected by microplate reader to calculate the survival rate.

Cell tube forming experiment: HUVEC cells were cultured to a good state, mixed with the supernatant of cell culture medium of HCT116 groups according to the above groups, added into a 24 well plate with matrix glue, cultured for $6 \mathrm{~h}$, took tube forming photos, and analyzed the length of tubules and junctions with Image $\mathrm{J}$ software.

Statistical analysis: SPSS 19.0 statistical software was used for data analysis. The measurement data were expressed as mean \pm standard deviation. The mean between the two groups was compared by independent sample t-test, and the counting data were tested by chi square test $(X 2 \nabla, P<0.05$, the difference was statistically significant.

\section{Results}

The mean value of serum YKL-40 in colorectal cancer group, precancerous lesion group and control group were (178.50 \pm 71.91$)$ respectively ug/L区(91.37 \pm 35.79$) \mathrm{ug} / \mathrm{L}$ and $(78.23 \pm 26.52) \mathrm{ug} / \mathrm{L} \otimes$ Colorectal cancer group (preoperative) was significantly higher than precancerous lesion group and control group ( $\mathrm{P}$ $<0.01)$. The precancerous lesion group was higher than the control group, but there was no significant difference between the two groups $(P=0.244)$.

Preoperative serum YKL-40 levels of colorectal cancer patients with different pathological stages and degrees of differentiation: preoperative serum YKL-40 levels of colorectal cancer patients with different pathological stages and degrees of differentiation are shown in Table 1. The preoperative YKL-40 level of colorectal cancer was related to pathological stage and degree of differentiation $(P<0.01)$.

Changes of serum YKL-40 in patients with colorectal cancer before and after operation: the level of YKL40 in 76 patients with colorectal cancer increased from (178.50 \pm 71.91$)$ before operation $\mu \mathrm{G} / \mathrm{L}$ decreased to $(166.13 \pm 68.17)$ at 7 days after operation $\mu \mathrm{g} / \mathrm{L} \otimes$ The level of YKL-40 in peripheral blood of 30 patients after operation was higher than that before operation. By paired t-test, there was no significant difference in the expression of serum YKL-40 before and after operation $(P=0.07)$.

The colon cancer cell line with high expression of YKL-40 was screened: the cell with the highest expression of YKL-40 among the three human colon cancer cell lines HCT-15, SW480 and HCT116 was screened by WB detection. Results as shown in Figure 1, the expression of YKL-40 was the highest in HCT116 cells. 
Validation of transfection effect: the interference efficiency of YKL-40 was verified by qPCR and WB detection. Results as shown in Figure 2, sirna3 was significantly down regulated compared with the noload group $(P<0.05)$, so sirna3 was selected for follow-up experiment.

CCK8 proliferation results: the proliferation of each group was detected by CCK8. The results are shown in Figure 3. The survival rate of the experimental group was $78.75 \%$, which was significantly lower than that of the control group $(P<0.05)$.

Results of cell tube formation: the results are shown in Table 2. Taking the length of tubules and the number of cross points as the comparison indexes of tube formation ability, there are significant differences in the two indexes between the experimental group, the experimental group + bevacizumab and the control group + bevacizumab compared with the control group $(P<0.05)$. Moreover, the inhibition of the two indexes in the experimental group + bevacizumab is more obvious than that in the control group + bevacizumab experimental group, However, there was no statistical difference ( $\mathrm{P} 0.17$ and 0.59 respectively). The inhibition of experimental group + bevacizumab was more obvious than that of experimental group, but there was no statistical difference ( $\mathrm{P} 0.116$ and 0.105 respectively).

Table 2

angiogenesis of HUVEC cells in each group

\begin{tabular}{|lllll|}
\hline & control & experiment & Control+ bevacizumab & Experiment+ bevacizumab \\
\hline Total Tube length & 2908 & 2462.67 & 2426.33 & 2202.33 \\
\cline { 2 - 3 } & 31.33 & 25 & 21.33 & 19.67 \\
\hline
\end{tabular}

\section{Discussion}

Colorectal cancer is occult and needs to go through a slow pathological process. It takes about 5-10 years from precancerous lesions to colorectal cancer [17]. However, clinically, up to $70 \%$ of patients are in the middle and advanced stage of tumor when they appear symptoms [18]. Therefore, understanding the changes of genes related to the pathogenesis of colorectal cancer and its molecular mechanism is of great significance for the early diagnosis and treatment of colorectal cancer. YKL-40 gene is located on chromosome 1q32.1 [19], encoding a highly conserved glycoprotein combined with heparin and chitin. It has important functions in cell proliferation, differentiation, survival and tissue remodeling, and is only highly expressed in highly active embryonic or adult cells. It was found that YKL-40 was highly expressed in peripheral blood and tumor tissues of patients with renal cell carcinoma and was associated with poor prognosis [20]. In prostate cancer and glioblastoma, overexpression of YKL-40 can induce cell proliferation, inhibit apoptosis and hinder cell differentiation [21, 22]. Our study also found that the peripheral blood YKL-40 of patients with colorectal cancer was significantly higher than that of precancerous lesions and perianal benign diseases. With the progress of the disease stage, the expression level of YKL-40 in serum gradually increased $(P<0.05)$. Although the difference between precancerous lesion group and perianal benign disease group did not reach statistical significance, the 
former also showed a high expression level. The results suggest that YKL-40 has an important relationship with the occurrence and development of colorectal cancer, and its expression level reflects the degree of tumor stage, which may be related to its prognosis, which needs to be confirmed by followup results.

The expression level of YKL-40 in 76 patients before and after operation was also detected. Different from the assumption of the study, the average level of YKL-40 in peripheral blood of patients with colorectal cancer did not decrease significantly 7 days after operation. The expression level of 30 cases was higher than that before operation. It is inferred that the reason may be that the tumor tissue was squeezed during the operation, resulting in the release of YKL-40 into the blood.

In order to reduce interference factors, the cell lines with high expression of YKL-40 were selected and divided into two groups. One group reduced the expression of YKL-40 gene by siRNA technology, and the other group was still in high expression state. Comparing the cell proliferation of the two groups, it was found that the survival rate of HCT116 cells in the YKL-40 high expression group was significantly higher than that in the YKL-40 low expression group, suggesting that the gene coding product has a positive regulatory effect on the proliferation of tumor cells, which is consistent with the previous research results [23].

The growth of solid malignant tumors needs the support of microvessels. YKL-40 can directly or indirectly induce tumor angiogenesis by stimulating the action of vascular endothelial growth factor, so as to promote tumor growth and metastasis [8]. In this study, cell tube forming experiment was carried out. Interfering with the expression of YKL-40 can significantly inhibit angiogenesis in vitro, which is consistent with the previous research results. It is confirmed that $\mathrm{YKL}-40$ can promote tumor microvascular formation.

Anti VEGF monoclonal antibody (bevacizumab) combined with chemotherapy is one of the standard treatments for advanced colorectal cancer, but the therapeutic effect of anti VEGF monoclonal antibody in patients with colorectal cancer is very different, and there is no appropriate index to screen people who can benefit from anti VEGF monoclonal antibody treatment. Saidl a et al. [17] found that anti VEGF monoclonal antibody could not inhibit endothelial cell angiogenesis induced by YKL-40 in vitro [18]. We compared the response of YKL-40 high expression group and low expression group to bevacizumab intervention. It was found that the ability of bevacizumab to inhibit angiogenesis in YKL-40 low expression group was stronger than that in high expression group. Although there was no statistical difference, it suggested that YKL-40 low expression group seemed to benefit more from the anti-tumor vascular effect of bevacizumab.

It can be seen that YKL-40 plays an important role in the development of colorectal cancer and the formation of tumor blood vessels. It is expected to become a new oncological marker of colorectal cancer and guide the precise treatment of colorectal cancer. Of course, we should also recognize that the expression level of serum YKL-40 is disturbed by a variety of diseases, and the detection method lacks homogenization standards. Only by strengthening quality control management can it be applied to clinic. 
In addition, this study only selected the HCT116 cell line with high expression of YKL-40, and only carried out the experiment of monolayer cells in vitro, lacking the comparison between multi cell lines and animal experiments. The role and mechanism of the gene in the development of colorectal cancer need to be further studied.

\section{Declarations}

\section{Ethics approval and consent to participate}

the study was approved by the ethics committee of Ningbo Medical Treatment Centre Li Huili Hospital (ethics approval No. ky2019pj035).

\section{Consent for publication}

Not applicable.

\section{Availability of data and materials}

The data used in the current study are available from the corresponding author on reasonable request.

\section{Competing interests}

The authors declare that there were no conflicts of interest.

\section{Funding}

This work was supported by Natural Science Foundation of Ningbo City(2019A610334)

\section{Authors' contributions}

SHY conceived and designed the study, supervised experiments, and wrote the manuscript. JS performed experiments, analyzed the data. YBZ analyzed the data and revised the manuscript. All authors have read and approved the final submitted manuscript.

\section{Acknowledgements}

Not applicable.

\section{Authors' information}

Shaohui Yang, Email:doctoryang1980@163.com.

Jie shen,Email:Asclepius@126.com.

Yibin Zhao,Email:10093463@qq.com. 


\section{References}

1. Dekker E, Tanis PJ, Vleugels JLA, Kasi PM, Wallace MB,Colorectal cancer.Lancet.2019;394: 1467-80.

2. Schreuders EH, Ruco A, Rabeneck L, Schoen RE, Sung JJY, Young GP, Kuipers EJ.Colorectal cancer screening: a global overview of existing Programmes.Gut.2015; 64:1637-49.

3. Wong SH,Yu J.Gut microbiota in colorectal cancer: mechanisms of action and clinical applications. Nat Rev Gastroenterol Hepatol.2019; 16:690-704.

4. Allen J, Sears CL.Impact of the gut microbiome on the genome and epigenome of colon epithelial cells: contributions to colorectal cancer development, Genome Med.2019;11: 11.

5. Brody H. Colorectal cancer. Nature.2015; 521: S1.

6. Keum N, Giovannucci E. Global burden of colorectal cancer: emerging trends,risk factors and prevention strategies.Nat Rev Gastroenterol Hepatol.2019; 16:713-32.

7. Arnold M, Sierra MS, Laversanne M, Soerjomataram I, Jemal A,Bray F. Global patterns and trends in colorectal cancer incidence and mortality.Gut.2016;66:683-91.

8. Strum, WB. Colorectal adenomas. N Engl J Med. 2016; 374: 1065-75.

9. Maida, M, Macaluso, FS, laniro G,Mangiola F, Sinagra E,Hold G,Maida, C,Cammarota G,Gasbarrini A,Scarpulla G.Screening of colorectal cancer: Present and future.Expert Rev Anticancer Ther.2017;17:1131-46.

10. Rehli M, Krause SW, Andreesen R. Molecular characterization of the gene for human cartilage gp-39 (CHI3L1), a member of the chitinase protein family and marker for late stages of macrophage differentiation. Genomics.1997; 43:221-25.

11. Ringsholt M, Hogdall EV, Johansen JS,Price PA,Christensen LH.YKL-40 protein expression in normal adult human tissues-an immunohistochemical study. J Mol Histol.2007;38: 33-43.

12. Wan G, Xiang L, Sun X,Wang X, Li H, Ge W, Cao F. Elevated YKL-40 expression is associated with a poor prognosis in breast cancer patients.Oncotarget.2017;8:5382-91.

13. Roslind A, Palle C, Johansen JS,Christensen IJ, Nielsen HJ, Mosgaard BJ. Prognostic utility of serum YKL-40 in patients with cervical cancer. Scand J Clin Lab Invest. 2020;80:687-93.

14. Shahy EM, Taha MM, Ibrahim KS. Assessment of YKL-40, lipid profile, antioxidant status, and some trace elements in benign and malignant breast proliferation. Mol Biol Rep. 2020;47:6973-82.

15. Faibish M, Francescone R, Bentley B, Yan W, Shao R. A YKL-40-neutralizing antibody blocks tumor angiogenesis and progression: a potential therapeutic agent in cancers. Mol Cancer Ther. 2011;10:742-51.

16. Shao R, Francescone R, Ngernyuang N, Bentley B, Taylor SL, Moral L,Yan W. Anti-YKL-40 antibody and ionizing irradiation synergistically inhibit tumor vascularization and malignancy in glioblastoma. Carcinogenesis.2014;35:373-82

17. Strum,WB. Colorectal adenomas. N Engl J Med,2016;374: 1065-75. 
18. Maida M,Macaluso FS,laniro G, Mangiola F, Sinagra E,Hold G, Maida C, Cammarota G, Gasbarrini A,Scarpulla G.Screening of colorectal cancer:Present and future.Expert Rev Anticancer Ther.2017;17: 1131-1146.

19. Rehli M, Krause SW, Andreesen R. Molecular characterization of the gene for human cartilage gp-39 (CHI3L1), a member of the chitinase protein family and marker for late stages of macrophage differentiation. Genomics.1997;43:221-5.

20. Normanno N,De Luca A,Bianco C, Strizzi L, Mancino M, Maiello MR, Carotenuto A, De Feo G, Caponigro F, Salomon DS.Epidermal growth factor receptor (EGFR) signaling in cancer. Gene.2006;366: 2-16.

21. Klein S, Levitzki A. Targeting the EGFR and the PKB pathway in cancer.Curr Opin Cell Biol.2009;21: 185-93.

22. Hideko S, Hikari B, Hiroaki KJ, Rina N, Tomonori O, Naomi ST, Hiraku S, Makoto S, Shinichi S, Tomomitsu M.YKL-40 Promotes Proliferation of Cutaneous T-Cell Lymphoma Tumor Cells through Extracellular Signal-Regulated Kinase Pathways.J Invest Dermatol.2020;140:860-8.

23. Saidi, A, Javerzat, S, Bellahcene, A, Vos JD, Bello L,Castronovo V, Deprez M, Loiseau H, Bikfalvi A, Hagedorn M. Experimental anti- angiogenesis causes upregulation of genes associated with poor survival in glioblastoma. Int J Cancer.2008;122:2187-98.

\section{Figures}
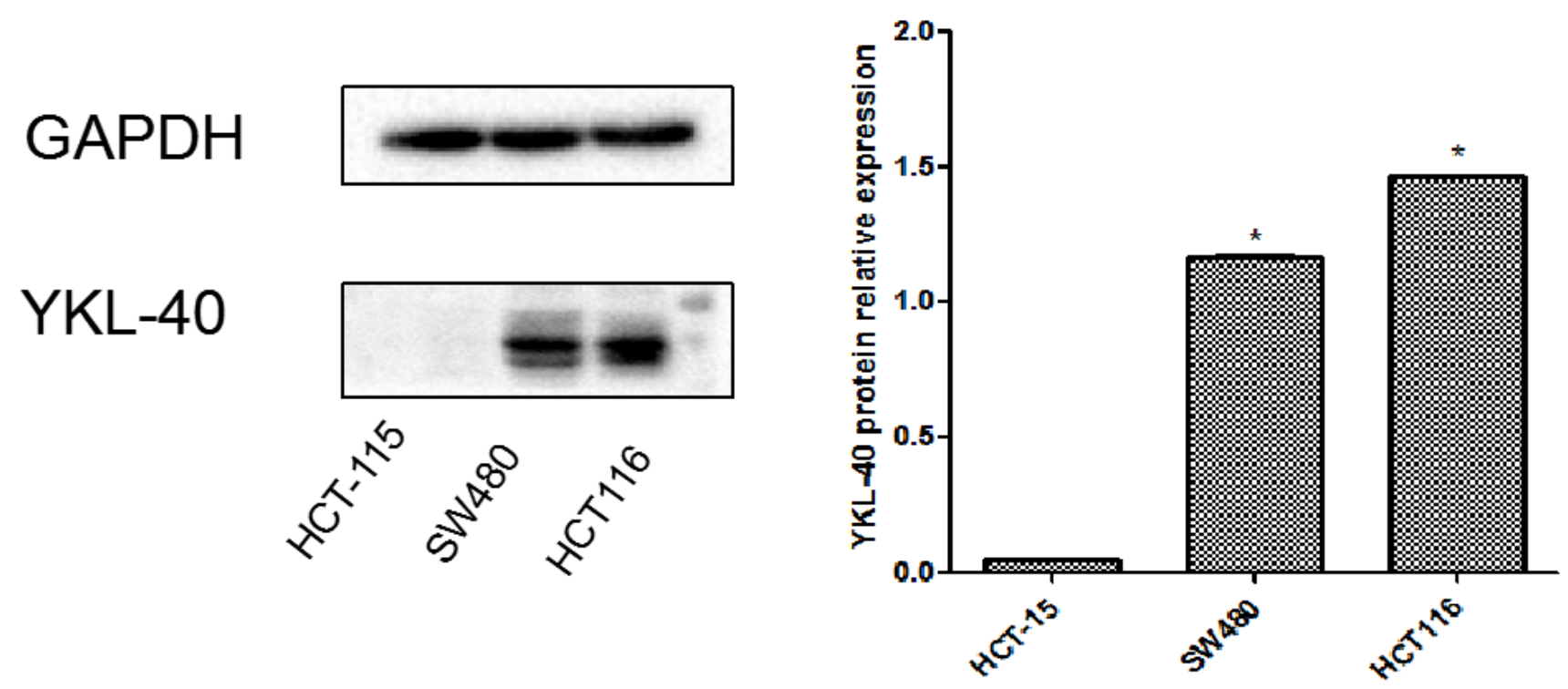

\section{Figure 1}

WB detection of YKL-40 expression in HCT-15, SW480 and HCT116 human colon cancer cell lines 




Figure 2

interference efficiency of YKL-40 verified by $\mathrm{qPCR}$ and WB 


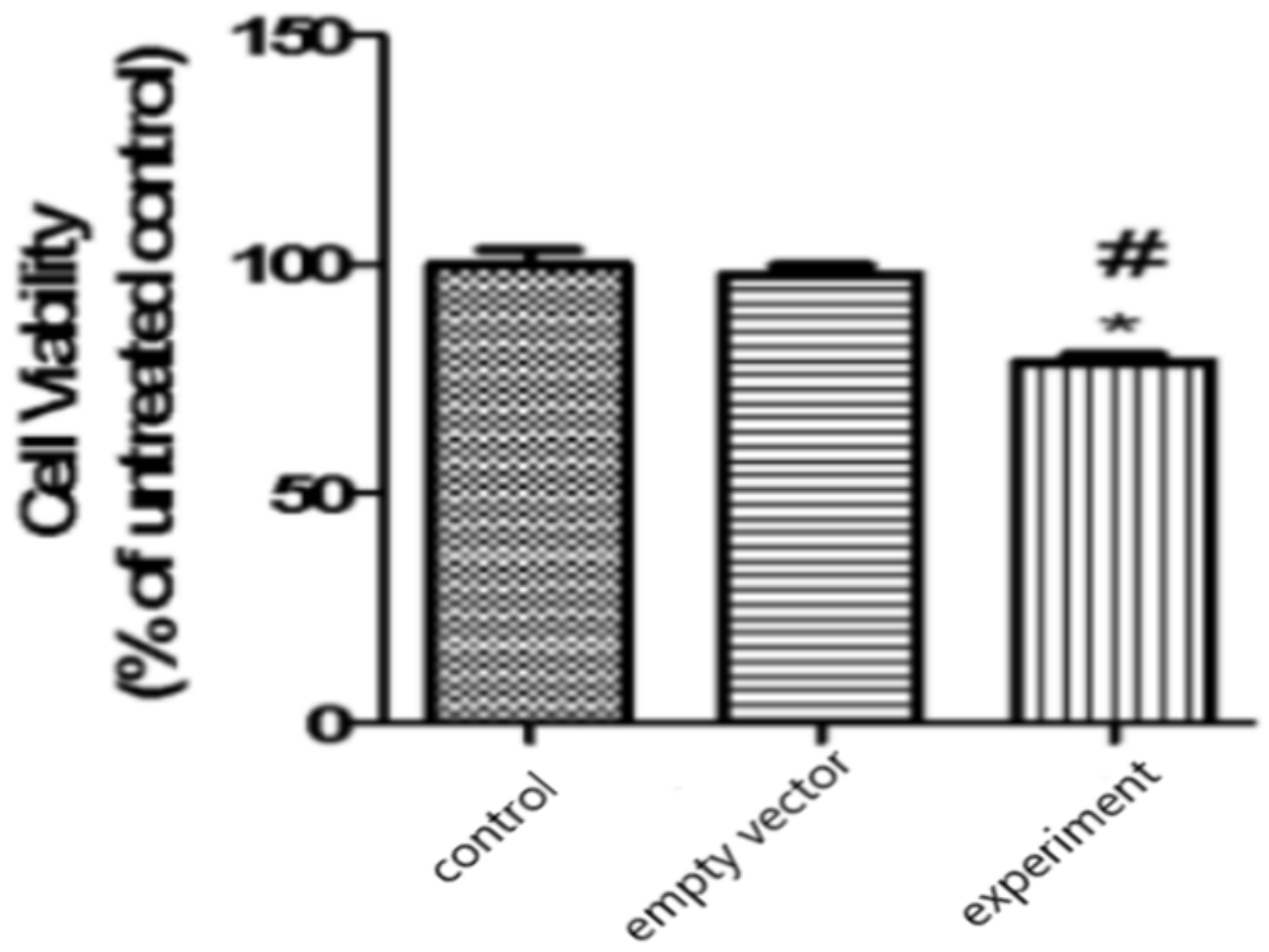

Figure 3

results of HCT116 cell proliferation 